\title{
On the Feasibility of a Moving Support for Surgery on the Beating Heart
}

\author{
A. L. Trejos ${ }^{1}$, S. E. Salcudean ${ }^{2}$, F. Sassani ${ }^{1}$, and S. Lichtenstein ${ }^{3}$ \\ 1 Department of Mechanical Engineering \\ 2 Department of Electrical Engineering \\ University of British Columbia, Vancouver, BC, Canada, V6T 1 Z4 \\ tims@ece.ubc.ca \\ 3 Department of Cardiovascular Surgery, St. Paul's Hospital, 1081 Burrard, \\ Vancouver, BC, V6Z 1Y6
}

\begin{abstract}
In this paper the use of a heart-tracking hand support is proposed to allow coronary artery bypass grafting surgery to take place on the beating heart. Requiring only a three-degree-of-freedom motion platform that tracks a point on the surgical site, this method eliminates the tissue damage associated with the use of physical heart stabilizers and provides a much cheaper alternative to six-degree-of-freedom masterslave systems.

To demonstrate the feasibility of such an approach, a motion platform with a motion range and frequency typical of coronary motion was designed and built. A task typical of suture placement was executed on this platform by twenty six subjects, while (i) the platform was stationary, (ii) the platform was in motion with the subjects' hands attached to it, and (iii) the platform was in motion but the subjects' hands were not attached to it. This system simulates the use of a motion tracking platform with perfect tracking performance. Mono and stereo vision systems were also mounted to the platform to provide subjects with a visually stable view. Accuracy and task completion time were measured.

Relative to the stationary platform case, only a $10 \%$ loss of accuracy and $40 \%$ increase in completion time were noted when the platform was in motion but the subjects' hands were attached to it. When the hands were not attached, a significantly higher $50 \%$ loss of accuracy and $100 \%$ increase in task completion time were noted. Task completion time was improved when a visually stable view was also provided, but accuracy results were inconclusive due to problems with the vision systems used.
\end{abstract}

\section{Introduction}

Coronary artery bypass grafting $(\mathrm{CABG})$ surgery requires complex grafts on the surface of the heart. While a bloodless anastomotic field can be easily achieved by a temporary coronary occlusion [2], achieving the motionless surgical field required for such delicate work is much more challenging and requires cardiopulmonary bypass with the use of a heart and lung machine. While performing the circulation and filtration of blood that allow the heart to be stopped, such a 


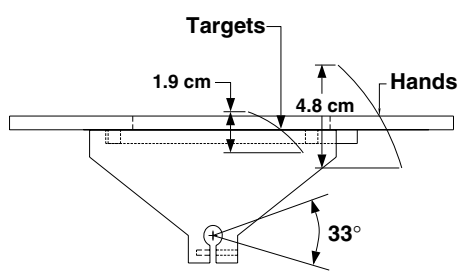

Fig. 1. Platform Motion

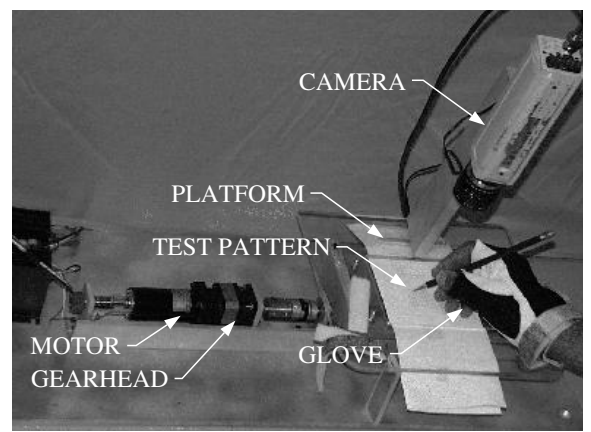

Fig. 2. Moving Platform

machine has many damaging effects on the patient's blood, and leads to high surgery costs and long recovery times [1].

Previous studies have been made to allow CABG surgery to be performed on the beating heart. The proposed methods work by stabilizing the surgical area on the surface of the heart, either by pressure on the tissue $[3,4]$ or by attaching an apparatus using suction $[5,6]$. The use of stabilizers that work by pressure have the disadvantage of allowing grafts to be performed only on the top surface of the heart. Stabilizers that work using suction have the disadvantage of damaging the heart tissue, even when used for short periods of time [7].

In principle, the master-slave robotic systems that have been developed to perform minimally invasive heart surgery $[9,10]$ could be modified to allow tracking of the heart motion by the endoscope and surgical instruments carried by the slave robot. The slave robot motion relative to the surgical site would follow the motion of the master robot, giving the user of the master robot the impression of working on a stationary surgical field. However, such a system would be extremely complicated, and likely would require at least six-degree-of-freedom systems for each of the master and the slave robots.

In this paper, the use of a motion platform that moves the hands of the surgeon in synchrony with the heart motion is proposed for CABG surgery. Such a motion platform would track the position of or the forces on a selected anastomosis site by using optical or mechanical sensors and a feedback control system, and would work in practice if (i) near-zero relative motion could be achieved between the moving platform and the surgical site, and (ii) surgeons could perform delicate tasks such as anastomosis on a moving surgical site as long as their hands are moved with the site.

The objective of this paper is to prove the latter point. Perfect tracking is simulated by having the operator perform a task on the same platform to which the hands are attached. The paper also aims to determine the need for a vision system that provides a stable view of the hands, tools and workspace.

If a system as proposed above did work in practice, it would have the following advantages: 


\begin{tabular}{|l|l|l|}
\hline Equipment & Brand $\backslash$ Model & Characteristics \\
\hline \hline Motor & Maxon $\backslash$ RE035-071-34EAB200A & 90 Watt DC, Stall Torque 1.1 Nm \\
\hline Gearhead & Sterling Inst. $\backslash$ S9117A-PG010 & Planetary, Ratio 10:1, single stage \\
\hline Camera & Philips $\backslash$ VC72505T & Color CCD \\
\hline Cameras & Pulnix $\backslash$ TM-545 & Monocrome CCD, 510 x 492 pixels \\
\hline Stereo Goggles & Keiser Electro-optics $\backslash$ VIM 500 & Color, 180000 pixels, 4:3 ratio \\
\hline
\end{tabular}

Table 1. Equipment details

- cardiopulmonary bypass and its damaging effects would be eliminated,

- damaging effects on heart tissue due to stabilization forces would be drastically reduced or eliminated,

- the surgeon's dexterity would be largerly preserved because the tools are manipulated directly without the loss of dexterity imposed by teleoperation,

- low cost relative to teleoperation systems, as only a 3 -DOF platform is required, as opposed to 6-DOF master and slave robots for each instrument.

A moving hand support that moves in synchrony with the heart was independently proposed recently in [8], where it was suggested that the heart motion be paced by the platform motion controller. Pacing the heart motion instead of following it can lead to large tracking errors and has clear clinical disadvantages.

\section{Experimental Setup}

\subsection{Moving Platform}

To test the ability of a human to perform a task on a moving target while the hands are being moved with it, an experimental platform was built. The motion of the platform roughly imitates the heart motion through an oscillatory rotation around a shaft. Figure 1 shows the vertical displacement in centimeters of the hand and the targets caused by a $33^{\circ}$ rotation of the shaft.

The hand of the subject can be attached to the platform by means of a glove fixed to it, so that it tracks the platform. To provide a guide for the task to be performed, a piece of paper having a suture target pattern (see section 2.2) can be inserted and fixed at a desired position. This simulates perfect tracking of the surgical site by the hand, since both the hand and the target are fixed to the moving platform. It is possible to attach one or two cameras to the platform so that the workspace can be seen either on a TV screen (1 camera, mono vision) or on a pair of goggles (2 cameras, stereo vision).

Figure 2 shows a photograph of the platform with one camera mounted on it. Table 1 shows the details of the equipment used in the system.

Figure 3 shows a diagram of the functional components of the system. The desired platform angle was generated by a PC as a pulse width modulated (PWM) signal. This was converted to a reference voltage for a standard PID motor controller loop. 


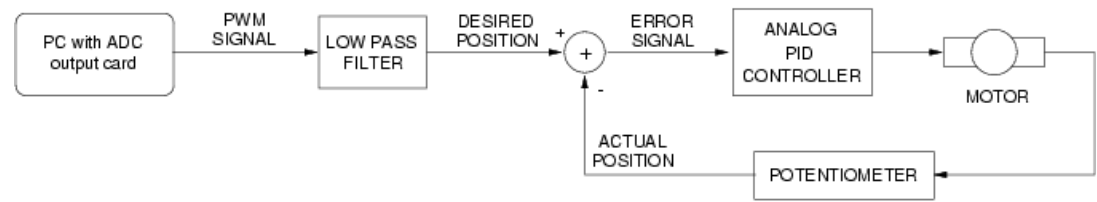

Fig. 3. Functional Components of the System

The original waveform used by the program was determined by measuring the motion of the medial coronary artery of a pig's heart which was then scaled to a human heart [6]. Figure 4 shows a typical plot of the actual motion of the targets as a function of time.

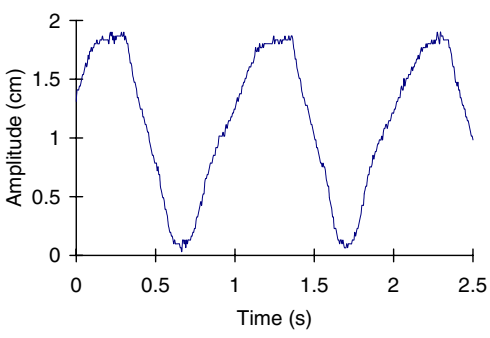

Fig. 4. Typical motion plot

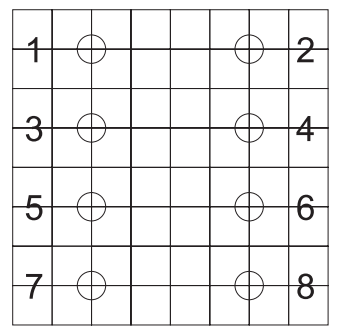

Fig. 5. Test pattern

\subsection{Task and Test Design}

The task performed simulates a simple suturing process. It consists of marking with a pen one single dot inside each of the circles of a pattern as seen in Figure 5, in the order shown by the numbers. The diameters of the circles, the grid spacing and the respective target size assigned for the four different sizes used are shown in Table 2.

\begin{tabular}{|c|c|c|}
\hline Target Size & Diameter $(\mathrm{mm})$ & Grid Spacing $(\mathrm{mm})$ \\
\hline \hline 1 & 3.43 & 4.76 \\
\hline 2 & 2.29 & 3.18 \\
\hline 3 & 1.73 & 2.38 \\
\hline 4 & 1.14 & 1.59 \\
\hline
\end{tabular}

Table 2. Test pattern dimensions

A total of seven different tests were performed, as follows:

1. Stationary platform and direct visualization of the workspace. This situation simulates suturing on an arrested heart. 
2. Stationary platform and visualization of the workspace on a TV screen. This test was added as a reference to Test 6 below.

3. Stationary platform and visualization of the workspace through stereo goggles. This test was added as a reference to Test 7 below.

4. Platform in motion and no attachment of the hand. This test simulates suturing on the beating heart without any stabilization help.

5. Platform in motion, hand attached to it and direct visualization of the workspace. This test simulates suturing on the surface of the beating heart with the hands being moved to track the surgical site.

6. Platform in motion, hand attached to it and visualization of the workspace on a TV screen. These are the same conditions as in Test 5, with the subjects seeing a stationary two-dimensional image of the surgical site.

7. Platform in motion, hand attached to it and visualization of the workspace through stereo goggles. To provide depth perception, two cameras and a pair of stereo goggles were used to produce a three-dimensional image.

The hand that was attached to the platform during tests 5 to 7 depended on whether the subject was left or right handed.

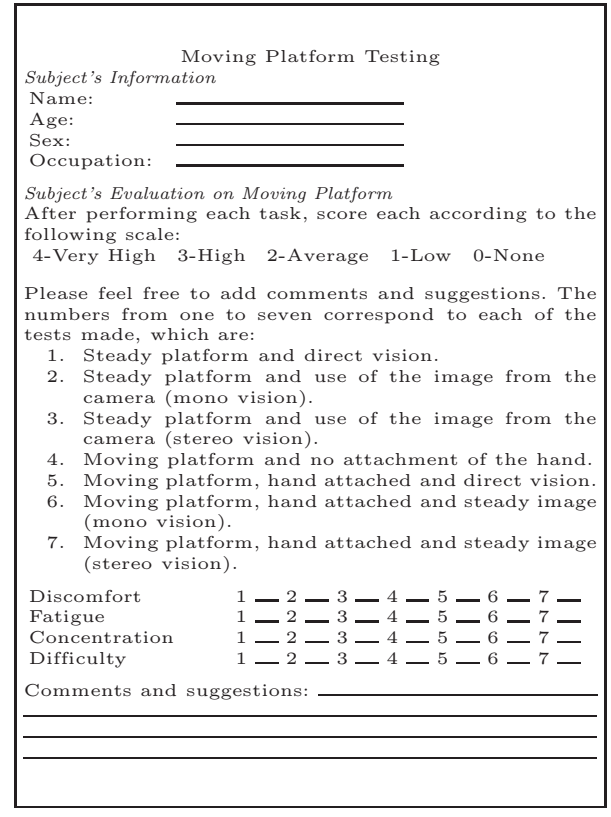

Fig. 6. Questionnaire

\begin{tabular}{|c|l|}
\hline Score & Characterisitcs of the mark \\
\hline \hline 6 & A neat dot inside the target \\
\hline 5 & $\begin{array}{l}\text { A smear inside the target or } \\
\text { one neat dot in contact with } \\
\text { the circle }\end{array}$ \\
\hline 4 & $\begin{array}{l}\text { A smear in contact with the } \\
\text { circle }\end{array}$ \\
\hline 3 & $\begin{array}{l}\text { A neat dot outside the circle } \\
\text { but inside the four quadrants } \\
\text { surrounding the target }\end{array}$ \\
\hline 2 & $\begin{array}{l}\text { A smear outside the circle } \\
\text { but inside the four quadrants } \\
\text { surrounding the target }\end{array}$ \\
\hline 1 & $\begin{array}{l}\text { A smear or a dot that is } \\
\text { outside the four surrounding } \\
\text { quadrants }\end{array}$ \\
\hline
\end{tabular}

Table 3. Accuracy scoring scale

\subsection{Testing}

Twenty six subjects were tested, comprised of three cardiac surgeons, one professor and twenty two graduate students. The age of the subjects ranged from 22 to 49 years of age. Seven were older than 30 years of age and nineteen were 
younger. Seven subjects were female and nineteen were male. Two of the subjects were left handed.

Although surgeons perform training exercises similar to the task performed, none of the other subjects had any experience in performing such tasks. For this reason they were allowed to practice as much as they felt was necessary. Each subject was told to perform the task as accurately and quickly as possible, giving greater importance to the accuracy than to the speed. Each of the subjects performed the seven tests in a random order.

After each test, subjects were asked to fill out the subjective questionnaire shown in Fig. 6.

Three of the subjects were asked to perform the test set an additional two times with the goal of determining whether there was a learning process involved when performing the task.

\subsection{Quantification of Performance}

The two factors measured to judge performance were task completion time and the accuracy and neatness of the mark made with the pen. To measure accuracy the scoring scale shown in Table 7 was used. If two marks were made, the one further away from the target was considered for scoring purposes.

\section{Experimental Results}

Table 4 shows the measured values for task completion time and accuracy for Test 1 . These values are averaged over the 26 subjects. The standard deviations are indicated.

\begin{tabular}{|c|c|c|}
\hline Target Size & Accuracy Score & Completion Time (s) \\
\hline \hline 1 & $46.6 \pm 2.4$ & $6.52 \pm 2.6$ \\
\hline 2 & $47.2 \pm 1.3$ & $6.04 \pm 2.0$ \\
\hline 3 & $47.3 \pm 1.4$ & $6.08 \pm 2.1$ \\
\hline 4 & $45.5 \pm 2.5$ & $6.31 \pm 2.2$ \\
\hline
\end{tabular}

Table 4. Average results for test 1

\begin{tabular}{|l|l|}
\hline 4 & Very High \\
\hline 3 & High \\
\hline 2 & Average \\
\hline 1 & Low \\
\hline 0 & None \\
\hline
\end{tabular}

Table 5. Scoring scale

For each subject, all measured values were normalized with respect to the test where the platform was stable and the same vision system was used. The graphs show the average results obtained by the twenty six subjects. The error bars show the standard deviation of the measurements from the average.

\subsection{Results Obtained from Tests with Direct Vision}

Figures 7 and 8 show the decrease in accuracy and increase in the task completion time, respectively, that result from a moving platform compared to a stationary platform for the four different target sizes (see Table 2). The results show that without the hand support, the accuracy decreases by more than $50 \%$ 
when performing the task on the smallest pattern size. When the hand is attached to the platform this reduction is less than $11 \%$, even for the smallest targets.

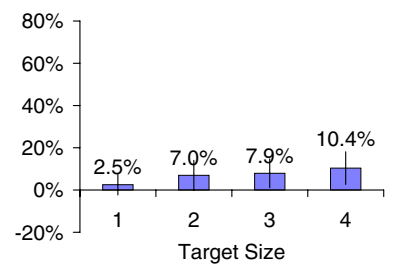

(a) Hand attached

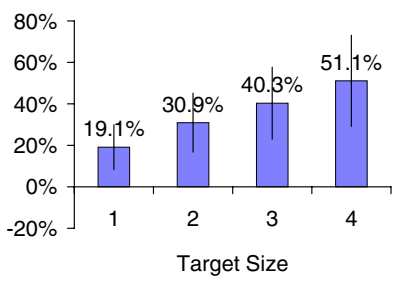

(b) Hand not attached

Fig. 7. Decrease in accuracy

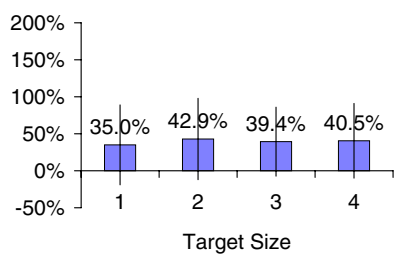

(a) Hand attached

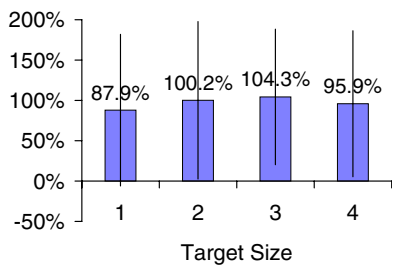

(b) Hand not attached

Fig. 8. Increase in task completion time

When the hand is attached and the platform is in motion, the average task completion time is increased by around $40 \%$ with respect to when the platform is stationary, independent of the target size. This shows a considerable improvement over unattached hand, where the increase in task completion time is doubled.

\subsection{Results from the Use of Different Vision Systems}

Further analysis was made to determine the advantages or disadvantages of using different vision systems to provide a steady image of the workspace. The results are compared to the test with direct vision in Figures 9 and 10.

These graphs show that the accuracy is reduced by approximately $15 \%$ for the smallest target using mono vision and $18 \%$ using stereo vision. These values are both greater than for direct vision. On the other hand, the stereo vision system produces the least increase in task completion time - less than $30 \%$ for all the target sizes. 


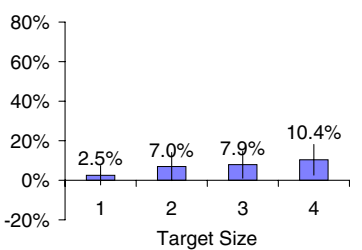

Direct vision

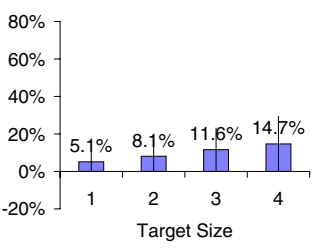

Stable 2D image

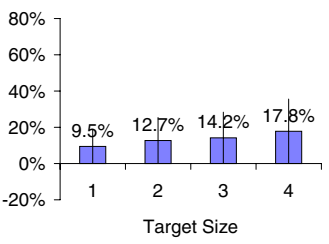

Stable 3D image

Fig. 9. Decrease in accuracy when using different vision systems

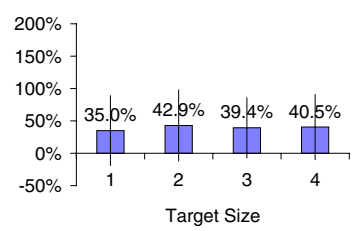

Direct vision

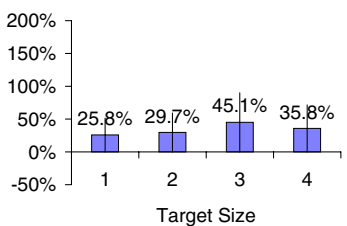

Stable 2D image

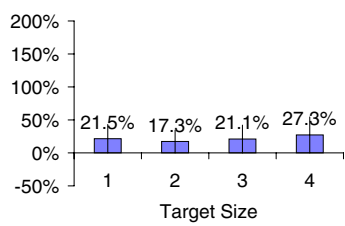

Stable 3D image

Fig. 10. Increase in completion time when using different vision systems

\subsection{Questionnaire Results}

The graphs in Figure 11 show the results of the subjective evaluation made by each test subject. The scale used by the subjects for scoring purposes is shown in Table 5. The test number shown corresponds to the list given in Section 2.2.

As can be seen from the graphs, subjects found the test with no hand support and the platform in motion (Test 4 ) to be the most uncomfortable, fatiguing and difficult one, as well as the one that required the most concentration. When the platform was stationary, the test with direct vision (Test 1) was perceived to be the easiest and simplest to perform. Looking at the image on the TV screen (Test 2) in 2D or with the goggles (Test 3) in 3D caused the task to be more difficult and to require higher concentration. In addition, the use of the goggles produced the greatest discomfort and fatigue.

These results are consistent with the results obtained when the platform was in motion (Tests 5, 6 and 7), although there is a general increase in all the factors due to the motion.

\subsection{Results of the Learning Process}

The results obtained when three of the subjects repeated the test two more times show that there is no general tendency for an increase in accuracy or decrease in task completion time for the second or third performances. In some cases there is improvement, but not in all the tests. 


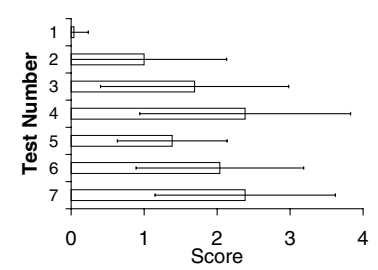

(a) Discomfort

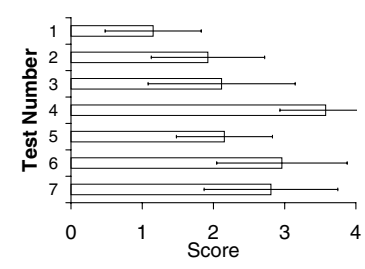

(c) Concentration

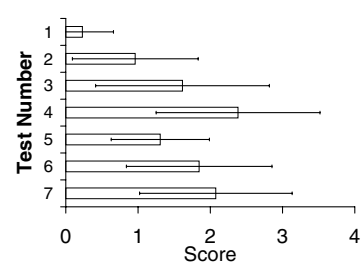

(b) Fatigue

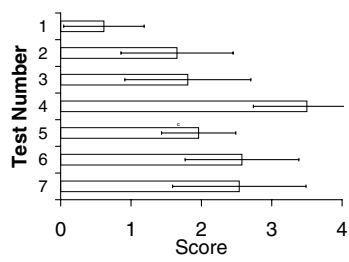

(d) Difficulty

Fig. 11. Questionnaire results

\section{Discussion}

The direct vision results demonstrate that when the hand moves in synchrony with the task space, there is a significant improvement in the accuracy achieved. Using a vision system to provide a steady image of the workspace and the hand did not further improve the accuracy of results; however, there are several factors that have to be taken into account when analyzing these results. The TV screen used in the 2D system is located in front of the subject, causing the workspace to be seen as a vertical surface instead of a horizontal one. In addition, there is lack of depth perception. When using the stereo goggles, although the 3D image provided depth perception, problems arose due to the low resolution of the image (17 pixels across smallest target) and the fact that the image was located at the edge of the depth of field of the camera.

Due to the nature of the surgical task, magnification of the workspace is a necessity. Without the use of a vision system, capable of following the motion of the heart, the surgical area would likely pass out of the field of view of the magnification apparatus. It is believed that if a clear and magnified stereo image is provided, the results obtained could be as good or better than those obtained when looking at the surgical area directly.

When analyzing the results obtained for task completion time, it was noticed that there was a high degree of variability among the subjects, as seen from the large standard deviations shown in the graphs. This variability was noticeable in all of the tests, including those performed when the platform was stationary 
(Table 4). The main cause of this variability is the random order in which the tests were performed - it was common for subjects to decrease completion time from the first tests to the last. This makes it difficult to obtain conclusive results from the graphs; however, the analysis was made based on the general tendency, showed by the average value obtained from all the subjects.

There was an increase in the task completion time when performing the task with the platform in motion regardless of the vision system used. This increase in time is considered to be much less than that required for the use of the heart and lung machine (approximately 80 minutes); therefore, the approach is still viable.

The increase in task completion time when using the stereo vision system was slightly less than when using the $2 \mathrm{D}$ system or direct vision. By offering a stable, 3D image of the workspace, the motion of the platform did not degrade performance as much as with the other vision systems. This agrees with the results of the questionnaire which indicate that the $3 \mathrm{D}$ image reduced the concentration requirements and perceived difficulty of the task.

The results from the questionnaire also indicate that there is a need to improve the design of the apparatus. The levels of discomfort, fatigue, concentration and difficulty experienced by the subjects when the platform was in motion can be reduced by a better design of the support and the vision system.

It is worth noting that there is no significant performance improvement to be realized by repeating the tests several times. This is demonstrated by the tests used to evaluate the learning process. Also, the ability to perform the tests was not influenced by surgical skill, as the performance of the three surgeons was very similar to the other, unskilled subjects.

\section{Conclusions and Future Work}

A new method based on a moving hand and/or tool support that tracks the motion of the heart has been proposed for performing coronary artery bypass grafting surgery on the beating heart. This method involves small or zero forces on cardiac tissue and could provide a low cost alternative to master-slave systems in offering a less invasive surgery, with reduced recovery time and secondary effects.

Twenty six subjects evaluated the feasibility of this approach by executing a task on a moving platform while their hands were attached to it. The situation simulated the use of a motion platform that tracks the surgical site perfectly. The experimental results show that it is possible to perform accurate tasks while the hands are being moved in synchrony with the workspace.

The results obtained when using the moving hand support are clearly improved as compared to those when it was not used. The average accuracy of the tasks was nearly unaffected (more than 90\%). Average task completion time was increased by approximately $40 \%$. Although our data show no improvement realized by providing a stationary image, the results could change significantly for the better if a high resolution stereo vision system were used. 
The need for a magnified image justifies the need for a vision system that follows the motion of the workspace. The vision system used should provide a stable stereo image with very good resolution and clarity.

Potential improvements to the apparatus include an ergonomically designed support that guides and supports the hand comfortably. Adjustments to allow the tool that is being used to also be attached and moved in synchrony with the heart would be beneficial. The main future work will involve evaluating methods to track the heart motion in real time and designing a mechanism that produces the required platform motion.

\section{Acknowledgments}

This study has been supported by the Canadian IRIS/PRECARN Network of Centres of Excellence, Project SAL. Special thanks to Terence Gilhuly for his helpful work and ideas.

\section{References}

1. J.W. Kirklin and B.G. Barratt Boyes, Cardiac Surgery: Morphology Diagnostic Criteria, Natural History, Techniques, Results and Indications. New York, USA: Churchill-Livingstone Press, 1993. 1089

2. Robert W. Emery, ed., Techniques for Minimally Invasive Direct Coronary Artery Bypass (MIDCAB) Surgery. Philadelphia, PA: Hanley and Belfus, Inc., 1997. 1088

3. Piet W. Boonstra, "Local immobilization of the left Anterior Descending Artery for Minimally invasive Coronary Bypass Grafting," Annals of Thoracic Surgery, Vol. 63, pp. S76-8, 1997. 1089

4. Guidant CVS Group, "Cardiovascular Products," 1998. http://www.guidant.com/cvs /product/vasoview.html 1089

5. Cornelius Borst, "Coronary Artery Bypass Grafting without Cardiopulmonary Bypass and without interruption of Native Coronary Flow using a novel Anastomosis Site Restraining Device ("Octopus")," Journal of American College of Cardiology, vol. 27, no.6, pp. 1356-64, May, 1996. 1089

6. Terence Gilhuly, "Optical and Physical Heart Stabilization for Cardiac Surgery," April, 1998. Master's Thesis, Department of Electrical Engineering, University of British Columbia 1089, 1091

7. David C. Youmans, Ethicon Endosurgery. Personal Communication. 1089

8. Paul W. Mayer, "Relative motion canceling platform for surgery," Feb. 15, 1999. U.S. Patent No. 5871017. 1090

9. Computer Motion "Robotic Surgical System Enabling Minimally Invasive Microsurgery," 1998. http://www.computermotion.com/old/zeus.htm. 1089

10. Tala Skari "The Cutting Edge: Heart surgery enters the age of robotics," Life, Special Issue, pp. 14-23, Fall, 1998. 1089 